\title{
Seeing Officiating as a Sociotechnical System - The Case for Applying Distributed Situation Awareness to Officials in Sport
}

\author{
Timothy J. Neville ${ }^{1,2(\bowtie)}$ and Paul M. Salmon ${ }^{1}$ \\ ${ }^{1}$ Centre for Human Factors and Sociotechnical Systems, \\ The University of the Sunshine Coast, Sippy Downs, QLD, Australia \\ timothy.neville@research.usc.edu.au, \\ psalmon@usc.edu.au \\ 2 Joint and Operations Analysis Division, Defence Science and Technology \\ Organisation (DSTO), Canberra, ACT, Australia
}

\begin{abstract}
Systems thinking, the notion that the unit of analysis should be the overall sociotechnical system, is a popular contemporary paradigm within cognitive ergonomics. Despite this, systems thinking applications have not yet emerged in the sporting context. We argue that systems thinking applications are required in sport, especially since sports systems are becoming more complex and technology dependent. Further, Officials in Sport (OiS), the controllers of the game, represent a critical but neglected research area. In this paper we explore whether the cognitive ergonomics model of Distributed Situation Awareness (DSA) can be applied to OiS systems and if DSA provides appropriate theoretical and methodological approaches to support future studies of Situation Awareness in OiS systems. The implications for future sport and OiS research applications are discussed and a research agenda designed to facilitate these applications is proposed.
\end{abstract}

\section{Introduction}

Professional sports have seen a noticeable increase in the use of technologies to support training, skill development, performance and decision making. When considering the use of technologies to support decision making, the greatest area of contention sits with the use of aids by officials in sports - umpires, referees, judges and stewards. Spectators, coaches, players, the media and sponsors place frequent requests for more accurate decisions by officials. While technology has enabled such requests to be met, the process of its introduction and incorporation into the game is a frequently debated topic $[1,2]$.

The success of decision aid technology in sport depends as much about the way the users of the technology, officials, adapt their modes of operation to the new technology as it does with the performance characteristics of the technology itself [3]. Systems thinking has the potential to enable optimum integration of technology for officials in sport (OiS). With the notion that the unit of analysis should be the overall sociotechnical system as opposed to the individuals or technology within it, systems thinking is a popular contemporary paradigm within cognitive ergonomics $[4,5]$. As an example, the approach is currently popular in the road safety context [6], with recent 
applications applying systems thinking to road design [7], crash analysis [8], young drivers [9], and specific issues such as driver distraction [10]. Despite its popularity, systems thinking applications have not yet emerged in the sporting context. Sports systems, particularly OiS, are becoming more complex and technology dependent, exhibiting the characteristics of complex sociotechnical systems and represent a critical but neglected research area [11].

Systems approaches to cognitive ergonomics are wide and varied; from sociotechnical systems theory [3], to cognitive work analysis [12] and cognitive engineering [13]. These approaches, however, have focused on traditional forms of systems thinking - system design [14] or system safety analysis [15]. By focusing on OiS and technology use, a systems approach has the opportunity to both examine the design of existing systems and also explore the primary taksof OiS - decision making. Neville and Salmon [11] have identified that a systems approach to Situation Awareness, specifically the theory of Distributed Situation Awareness (DSA) [16], has applicability to both the design of the OiS system as well as their primary role to make decisions. DSA purports that situation awareness (SA), or understanding of 'what is going on' [17] is held by the overall sociotechnical system comprising human (officials) and nonhuman agents (e.g. pitch markings). An officials activates their awareness in the system through interactions with other agents [16, 18].

In this paper we argue that DSA provides appropriate theoretical and methodological approaches to support systems design and analysis for the OiS system. We explore this through elaborating on the six theoretical tenets of DSA [16] and demonstrating their applicability to the OiS system through examples from professional sport and through extracts from a DSA analysis of twoOiS systems. The implications for future sport and OiS research applications are discussed and a research agenda designed to facilitate these applications is proposed.

\section{A Systems Approach to Situation Awareness}

Traditional models of SA have taken an information processing based approach, where individuals or teams are said to have SA residing as knowledge within their mind $[17,19]$. The information processing model proposed the widely incorporated definition that SA is the perception of elements in the environment, comprehension of their meaning and projection of their future state [17]. Contrasting the information processing concept of SA is the ecological systems approach; that SA is a property of the system and activated through interactions between different agents in the system. Described as 'externally driven consciousness,' [20] an ecological systems approach allows for the unit of analysis to move from what is going on in one's mind to the way human and non-human agents interact in the system. Within the systems perspective of SA, DSA has been identified as the most applicable concept to be applied to OiS [11].

DSA is defined as "activated knowledge for a specific task within a system at a specific time by specific agents." [21], That is, humans or non-human agents use cues in the environment to activate relevant knowledge to enable them to complete a given task or goal. DSAsupports the distributed cognition movement [22], in that SA, like cognition, can be considered a property of the system as well as the individual. SA is 
the glue that holds the sociotechnical system together - it is more usefully viewed as residing in the interactions between agents rather than in the agents themselves. The unit of analysis is therefore the system and the way the agents use the awareness, which is distributed, to complete their goals or tasks.

To distinguish DSA from both distributed cognition as well as other established SA models, Stanton et al. [16] proposed six tenets as the basis of DSA. The tenets, in abbreviated form are:

1. "SA is held by human and non-human agents.

2. Different agents have different views on the same scene

3. Whether or not one agent's SA overlaps with that of another depends on their respective goals

4. Communication between agents may be nonverbal behavior, customs, and practice.

5. SA holds loosely couples systems together.

6. One agent may compensate for degradation in SA in another agent." [16]

The set of tenets provide a clear view of how SA is distributed with in a sociotechnical system. As such, the tenets allow for systems to be conceptually tested to identify if they are suitable for analysis using DSA.

\section{Situation Awareness, Sport and Officials}

To date there has been little exploration of SA in sport [17]. Of the sports applications presented in the literature, Macquet and Stanton [23], using the foundation of DSA, examined the compatibility of SA between an athlete and coach in Hammer Throwing and Rowing. De Keukelaere et al. [24], meanwhile, applied Shared SA [17] to examine if different Handball team members shared knowledge and understanding through the course of a game. Both applications focus on the way individuals operate either with other individuals or in a team situation. Within the OiS domain of sport there have beenno previous applications of SA models or methods [11].

Whilst OiS appears to be an appropriate domain in which to adopt the DSA perspective, it is important to confirm that DSA is applicable in this context; that is, do the six DSA tenets actually apply in OiS systems? The aim of this paper is to answer this question.

As it would be impractical to consider all forms or types of OiS systems due to the size of the data set (all sports) and the various forms of officials we will, instead focus each tenet on professional fast ball sports (such as various codes of football; tennis; baseball; cricket and rugby). In fast ball sports officials operate in teams and are augmented by technology such as communications systems, video review and goal line technologies, in order to aid adjudications through the course of a game.

\subsection{Tenet One - SA is Held by Human and Non-human Agents}

At the heart of DSA is that individuals and teams in sociotechnical systems cannot conduct their task, role or goal in the system without being able to interact with nonhuman agents. Non-human agents are either technological agents or various forms of 
artefacts in the system (such as physical, geographical, audible or visual). Examining first various forms of football (soccer American, Australian, Gaelic) or rugby (Rugby Union, 7's, Rugby League or Touch) each sport has a consistent use of a non-human agent - the whistle. Arguably a technological agent, the use of the whistle also introduces audible artefacts into the system. This tenet purports that both human and non-human agents 'hold' SA - that is, each provides a portion of awareness that underpins the systems DSA.

Certainly technologies such as video review and goal line technologies hold SA as they provide an overview of play for officials to support decision-making - they provide SA to facilitate officiating the game. Other non-technological agents provide an interesting point of discussion.

Does, for example, the umpire's whistle hold SA? The use of the whistle is to draw attention, to signal that something has occurred and to then act accordingly. When considering professional instances of fast ball sports, games are played infront of large crowds, often in excess of 50000 people. In these instances the role of whistle is magnified as the voice of the official can be considered part of the collected 'noise' of the game. While not necessarily a linear process, the interactions between players and officials is facilitated through the whistle. That is, players will only alter their behavior as a consequence of the whistle distributing a 'whistle' sound into the environment. Hence, a players awareness is activated through a transaction of SA held within the whistle (through the sounds it generates).

\subsection{Tenet Two - Different Agents have Different Views of the Same Scene}

A key tenet of DSA is the notion that SA is compatible; different agents hold different views on the same situation. The distinct portions of SA connect together to form the system's DSA. System design should therefore strive to support these different pictures and also connect them together. It is apparent that officials have different perspectives on the game to one another. Moreover, OiS systems could not function without compatible SA, since often the different views are brought together to arrive at the correct decision.

Initially, different views on the same scene invokes ideas of geographical distance; that based on an officials geographic position their 'view' of the scene will be different to that of another official in the system. Any team based OiS system operates with such arrangements. Within soccer referees and assistant referees have considerably different views based on their geographical location; indeed, decisional accuracy can be compromised based on these different geographical positions [25]. Similar instances exists in all forms of rugby with touch judges having a physical view perpendicular to that of the central referee.

Differing views of the same scene by different agents also exist in instances where geographic location is similar due to the use of technology. Cricket has introduced an infrared camera to identify 'hot spots' on the player and their equipment [26]. Using infrared imaging, the camera identifies when a player's bat hits the ball or when the ball hits a players clothing or protective equipment. Situated at 'square leg' at either end of the pitch, the camera's geographical perspective is extremely similar to that of the 
'square leg' umpire. Nevertheless, its ability to see light in the infrared spectrum provides it with a different view of the same scene compared to other agents in the system.

\subsection{Tenet Three - Whether or Not One Agent's SA Overlaps with that of Another Depends on Their Respective Goals}

The information processing perspective of SA proposes that Shared or Team SA is overlapping individual SA [27, 28]; that all members of a team have the same awareness of the system. Tenet three challenges thispersepective, stating that any set of agents' SA will only overlap based on the goals of each agent. In DSA, the appropriate time for SA to overlap is when the goals are similar or identical. If the goals are different then there is no need for the awareness of each agent to overlap. In fast ball sports, teams of officials exist due to the complexity of the game and subsequently, each official has different roles, goals and tasks to perform. The system has been designed for agents to have differing goals in order to achieve the most accurate decision possible.

In Australian Rules Football (AFL), for example, in response to the increased professionalism, athleticism and pace of the game the umpiring team now consists of 10 umpires $^{1}$ performing four broad roles(called disciplines) [29]. AFL is a fast, explosive, possession based, contact sport where two teams of 18 players compete through scoring points at either end of on an oval shaped field over $150 \mathrm{~m}$ in length. The pace of the game combined with the size of the playing field dictates that a central field umpire will be unable of have the same awareness as a boundary 'touch' umpire. The central umpire is required to adjudicate the majority of rule breaches in the field of play, the boundary umpire determines when the ball leaves the field of play. Their respective goals and awareness overlap in the few instances when the ball does leave the field of play: the boundary umpire signals the ball has gone out, the central field umpire is then required to acknowledge that the ball has 'gone out,' the two umpires then execute the procedure for returning the ball back to play. In the out of bounds process described the acknowledgement and then execution of procedures by both umpires demonstrates that each agents SA overlapped based on the goal of returning the ball to play.

Further, counter to the information processing perspective of overlapping Shared SA, consider the following example from soccer. Imagine a football referee and assistant referee officiating in the corner of a soccer pitch [25]. If the overlapping Shared SA model holds than the referee and assistant referee would share the same awareness of the situation. There would be, therefore, no need for the assistant referee to make any decision as the central referee has the same awareness and has seniority on

\footnotetext{
${ }^{1}$ Currently an AFL umpiring team consists of 10 umpires in three disciplines performing four roles Three Field Umpires adjudicate on the majority of rule breaches, four Boundary Umpires determine when and how the ball leaves the playing field, two Goal Umpires adjudicate the type of score on scoring plays and one Field Umpire acts as an emergency umpire. In the 1970's there were five umpires in the team - one Field, two Boundary and two Goal.
} 
the field. In reality, both hold a different perspective on the same situation and the resolution of this into a shared understanding (and decision) represents an important part of SA development - effectively how system actors transact different but compatible SA and achieve consensus.

\subsection{Tenet Four - Communication Between Agents may be Non-verbal Behavior, Customs, and Practice}

Tenet four relates to the transactions in awareness that occur between different agents, arguing that they can be achieved through non-verbal communications, customs and practice. For instance, Hutchins' famously showed that one such norm or practice was the way in which a cockpit remembers speed [22]. Within DSA, customs, norms and especially non-verbal behavior not only hold forms of cognition in the system but are also responsible for transaction of SA between agents in the system. The OiS system in itself but also as part of the broader system of the game relies heavily on non-verbal behaviors, customs and practices.

While such examples are present in all fast ball sports to varying degrees, tennis provides two clear examples of non-verbal communication being used to communicate to other agents. The first, also common to fast ball sports, is the use of hand signals to signify a breach of the rules. The linesperson, on noticing a ball landing outside the accepted area of the court, raises the appropriate arm to signify the ball is long. The non-verbal behavior acts as a form of communication to the chair umpire, players and spectators. Of course, the hand signal occurs in conjunction with verbal communications. Second, with professional tennis' embrace of technology, the 'cyclops' machine releases an audible chime when a player faults a serve. Both examples identify two instances when non-verbal communication is used to communicate between agents in the system.

A distinguishing feature of non-verbal communications in OiS is that they not only communicate between agents in the OiS system but also communicate to entities outside of the system. A referee's whistle, an umpires hand signal, a thrown flag all provide awareness to players, coaches, spectators and journalists that the situation as changed; highlighting an often over looked role that officials perform in sport.

\subsection{Tenet Five - SA Holds Loosely Coupled Systems Together}

Tightly coupled systems are those that are highly interdependent [30]; that is, changes to components impact other components across the system. Loosely coupled systems, on the other hand, have fewer dependencies between components meaning they can absorb changes with little impact on other components across the system.

According to Stanton et al. [16] DSA holds loosely coupled systems together. This tenet is not straightforward in its applicability to officials in fast-ball sports, primarily because it is not entirely clear which state OiS and sports systems operate in.

It may be that OiS systems dynamically shift between loose and tightly coupled states. In fast ball sports the OiS system may be loosely coupled during normal phases of play (as individual officials fulfill their own specific roles); however, it may 
transition to a tightly coupled state in complex scenarios that require coordination and teamwork (e.g. penalty decisions, red card decisions). If this is the case, not only does DSA hold the loosely coupled system together, it facilitates the transition between loose and tight coupling. This shifting of the system's coupling is a key research area, not only in sports and OiS, but in complex systems generally.

A second issue is that the coupling status may be dependent on the sport under consideration. For example, it may be that some fast ball sports are tightly coupled (e.g. tennis) whereas others are loosely coupled (e.g. Golf). In addition, individual or small team fast ball sports such as cricket might be considered a loosely coupled system; however the coupling of the officials is tight. Again the implications are interesting and this characteristic of sport and OiS systems highlights key research questions. For example, are the characteristics of DSA different in the loosely coupled cricket system compared to the tightly coupled cricket officials system?

The influence officials have on systems' coupling represents a key future research direction, as does the extent to which DSA holds loosely coupled sports systems together. Rather than DSA holding loosely coupled system together, is DSA actually part of the coupling mechanism; that any coupling of the system, loose or tight, is facilitated with the distribution of SA through the system?

\subsection{Tenet Six - One Agent May Compensate for Degradation in SA in Another Agent}

Redundancy is an important design consideration in safety critical systems, if one part of the system fails or degrades than another part of the system should be capable of compensating for the degradation. The loss of SA by agents in a system has been identified as the cause of incidents in safety critical systems such as many transport systems $[31,32]$. Tenet six draws these two ideas together, proposing that DSA enables other agents in the system to compensate for degradation in SA in another agent, providing redundancy in the system.

In the OiS context, this redundancy is important for distributed decision making. In fast ball sports the OiS system has a number of examples where one agent's SA is used to compensate for when another agent's SA degrades. The clearest example of this is the use of decision review systems, whether it be instant replay (MLB, AFL, NFL), Hawkeye (tennis, cricket) or centralized score or play review centers (MLB, NHL, NFL or Rugby League). These systems are designed to remove the proverbial 'howler' or blown call from the game. Errors in decision making by the one officials can be compensated by other agents (the review system). Similarly, in soccer linesmen are used to make offside decisions where the referee has insufficient SA to do so. While there has not been any research to identify if the decisional error in officials was caused by a degradation in SA, one could postulate that, for example, a baseball umpire might have degraded SA when a runner is tagged because the tag was not visible to him, or in football (all codes) referees or umpires may have degraded SA due to physical or cognitive fatigue or for the simple fact that a player was in their way. These systems are now designed to enable compensation to occur when degradation happens. 


\section{DSA Applicability to Officials in Sport - Initial Findings}

Through mapping the theoretical tenets of DSA to professional fast ball sports we have shown not only that DSA provides a new and novel way of describing officials in sports, but also that there is a conceptual fit between the model (DSA) and the domain of study (OiS). To further demonstrate the applicability of DSA to OiS we provide initial findings generated from two of the aforementioned fast ball sports soccer and AFL.

For the results from soccer we have taken a conceptual example of a foul situation in the corner area. The selection of this example was motivated by the results in [25], which identified that decisional accuracy decreases in this part of the soccer pitch. Figure 1 shows one of the key outputs within the DSA model, an indicative social network of a soccer OiS system. We see for this stand-alone instance there are a number of social actors in the system: players, referees, assistance referees, crowd, voice communications and equipment; all contributing to the systems' SA. Non-verbal communication has occurred through the assistant referees flag being used to provide awareness to the central umpire. The ground markings, as a non-human agent, provide artefacts which depict positional situation awareness to other agents. While, mentioned previously, the referee and assistant referee have different views of the same scene but also, for this particular instance, have unique but compatible overlapping SA. The DSA model will be able to identify the different perspective of each actor for this decision.

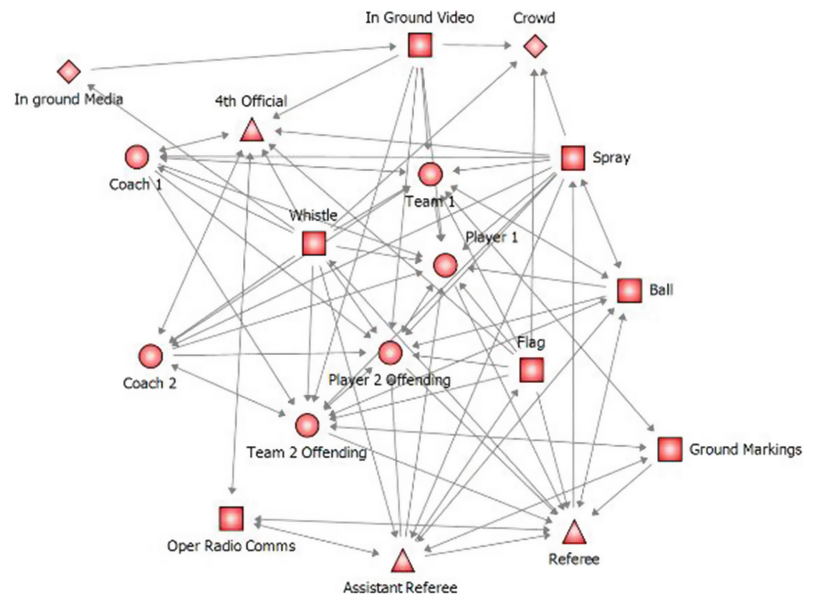

Fig. 1. Indicative Social interaction network for Soccer in the 'corner' area of the field. Arrows indicate the direction of interaction, triangle - officials; circle - player; square - technology agents; diamond - other actors.

The second example comes from AFL and demonstrates the nature of communications used to maintain DSA.

We have transcribed the communications the field umpires in three game from the 2014 AFL Premiership Season. AFL umpires wear a communications pack which 
enables them to constantly communicate to the other umpires. Within the recordings we identified audible communications instances: the use of whistle, and spoken words or phrases, which were projected through the course of a game. Our findings, presented in Table 1, demonstrate that within a game of AFL, field umpires will communicate through technology to other umpires $27.2 \%$ of the time, with $72.8 \%$ of the communication occurring between the umpire and players. In essence, normal communication is more dominant than technology enabled communication.

Table 1. Breakdown of normal and communication technology enabled communication from AFL field umpires.

\begin{tabular}{l|r|l|l|l|r}
\hline & \multicolumn{2}{l}{$\begin{array}{l}\text { Normal } \\
\text { communication }\end{array}$} & \multicolumn{2}{l}{$\begin{array}{l}\text { Technology enabled } \\
\text { communication }\end{array}$} & Total \\
\hline Game 1 Total & $\mathbf{1 4 1 3}$ & $69.61 \%$ & $\mathbf{6 1 7}$ & $30.39 \%$ & $\mathbf{2 0 3 0}$ \\
\hline Quarter 1 & 390 & $71.69 \%$ & 154 & $28.31 \%$ & 544 \\
\hline Quarter 2 & 373 & $70.51 \%$ & 156 & $29.49 \%$ & 529 \\
\hline Quarter 3 & 341 & $68.06 \%$ & 160 & $31.94 \%$ & 501 \\
\hline Quarter 4 & 309 & $67.76 \%$ & 147 & $32.24 \%$ & 456 \\
\hline Game 2 Total & $\mathbf{1 3 7 7}$ & $75.58 \%$ & $\mathbf{4 4 5}$ & $24.42 \%$ & $\mathbf{1 8 2 2}$ \\
\hline Quarter 1 & 310 & $70.94 \%$ & 127 & $29.06 \%$ & 437 \\
\hline Quarter 2 & 319 & $75.77 \%$ & 102 & $24.23 \%$ & 421 \\
\hline Quarter 3 & 393 & $77.51 \%$ & 114 & $22.49 \%$ & 507 \\
\hline Quarter 4 & 355 & $77.68 \%$ & 102 & $22.32 \%$ & 457 \\
\hline Game 3 Total & $\mathbf{1 5 4 4}$ & $73.49 \%$ & $\mathbf{5 5 7}$ & $26.51 \%$ & $\mathbf{2 1 0 1}$ \\
\hline Quarter 1 & 384 & $72.32 \%$ & 147 & $27.68 \%$ & 531 \\
\hline Quarter 2 & 388 & $76.38 \%$ & 120 & $23.62 \%$ & 508 \\
\hline Quarter 3 & 394 & $70.99 \%$ & 161 & $29.01 \%$ & 555 \\
\hline Quarter 4 & 378 & $74.56 \%$ & 129 & $25.44 \%$ & 507 \\
\hline Total & $\mathbf{4 3 3 4}$ & $72.80 \%$ & $\mathbf{1 6 1 9}$ & $27.20 \%$ & $\mathbf{5 9 5 3}$ \\
\hline
\end{tabular}

The results show how technology enabled communication builds upon the established mode of communication in umpire. The technology enabled communication provided the umpire greater information on the current situation such as which umpire is in control, which part of a ground an umpire needs to be, which players might need closer attention or, most importantly, that a rule infringement had occurred. The results indicate that communication equipment is used to facilitate the distribution of SA within the AFL OiS system. These 'transactions' in awareness are critical for maintaining DSA [33].

The breakdown on the use of communications equipment identifies that quantity of communications occurring between umpires in the game. The number of communications occurring between the members of the umpire team suggests that the constant open communications allows the team to perform their tasks more efficiently. Efficencyis achieved by the increase in the provision of situation relevant information. Potentially, the communication technology enables the system to be more tightly 
coupled, holding it together and reducing the likelihood of the degradation of the system's SA. This aligns with the notion that DSA and its mechanisms (e.g. transactions) act as the glue that holds sociotechnical systems together.

\section{Conclusions and Future Research Directions}

We have demonstrated, through the examining the six DSA tenets in relation to fast ball sports officials, that DSA provides an appropriate theoretical and methodological framework for understanding and optimizing OiS performance. Specifically, in presenting an exemplar social network and frequency statistics on the use of technology we show the complexity and impact non-human agents have in officiating. The absence of DSA applications confirms its potential as a new paradigm for sports ergonomics.

By studying OiS we anticipated that the DSAconcept of unique and compatible awareness can be tested in the field of organized sport. Further exploring OiS, whose role is to make decisions, may determine if SA is maintained and remains compatible over time as the consequences of decisions propagate through the system. In applying DSA to OiS, issues relating to the way officials train, their inter and intra team communications, the transfer of information and decision making can be identified and strategies for improvement suggested.

To realize the potential DSA offers, research should address several areas. Applying DSA to differing OIS will enable researchers to capture what constitutes DSA in a whole-of-officials context. Examining the DSA of multiple sports will determine if the current model is adequate or if extensions are needed, such as tenet five. Does DSA generalize across all OiS systems or is it specific to one sport only? We have identified that within the category of fast ball sports the tenets of DSA conceptually fit the OiS system, however, when applied to a specific sport do nuances in the sport require models to be extended to adequately capture the SA? If the OiS system is loosely coupled does DSA hold it together? An example of the possible insights which can be gained from applying the DSA model to the OiS system is seen in Fig. 1. Accumulating further standalone instances within a game, identifying changes of SA over time or geographic position (does SA degrade?) are all fields of enquiry which could be explored.

Previous DSA applications have proven useful for identifying measures to optimize system performance (e.g. [33]). Once an understanding of OiS system's DSA is achieved, research should examine the consequences of adding or removing specific agents from the system. We have demonstrated that there has been an increased role of technology in the OiS system, exploring the differences between instantiations of the system which have or do not have specific technologies offers potential impact to practitioners. For example, in Fig. 1, how does each actor's activation of SA change if 'Open Radio Comms' node was removed from the system? Do OiS performance metrics (such as decisional accuracy) increase or decrease? Can the impact new technology has on the system be predicted? Does the task of officiating a game become more or less complex? This is likely to become more important given the increasing use of technology in professional sports. DSA provides an appropriate theoretical and methodological framework to answer these questions. A notable flaw of current OiS 
system design is that new technologies are being introduced without examining these questions. Cognitive ergonomics practitioners should be working in this space, answering these questions. It is our opinion that the DSA model provides suitable tools for this endeavor; providing an exciting new paradigm for Cognitive Ergonomics.

\section{References}

1. Collins, H.: The philosophy of umpiring and the introduction of decision-aid technology. J. Philos. Sport 37(2), 135-146 (2010)

2. Collins, H., Evans, R.: You cannot be serious! Public understanding of technology with special reference to "hawk-Eye". Pub. Underst. Sci. 17(3), 283-308 (2008)

3. Clegg, C.W.: Sociotechnical principles for system design. Appl. Ergon. 31(5), 463-477 (2000)

4. Reason, J.: A systems approach to organizational error. Ergonomics 38(8), 1708-1721 (1995)

5. Wilson, J.R.: Fundamentals of systems ergonomics/human factors. Appl. Ergon. 45(1), 5-13 (2014)

6. Salmon, P.M., Lenné, M.G.: Miles away or just around the corner? Systems thinking in road safety research and practice. Accid. Anal. Prev. 74, 243-249 (2015)

7. Cornelissen, M., et al.: Assessing the 'system' in safe systems-based road designs: using cognitive work analysis to evaluate intersection designs. Accid. Anal. Prev. 74, 324-338 (2015)

8. Newnam, S., Goode, N.: Do not blame the driver: a systems analysis of the causes of road freight crashes. Accid. Anal. Prev. 76, 141-151 (2015)

9. Scott-Parker, B., Goode, N., Salmon, P.: The driver, the road, the rules... and the rest? A systems-based approach to young driver road safety. Accid. Anal. Prev. 74, 297-305 (2015)

10. Young, K.L., Salmon, P.M.: Sharing the responsibility for driver distraction across road transport systems: a systems approach to the management of distracted driving. Accid. Anal. Prev. 74, 350-359 (2015)

11. Neville, T.J., Salmon, P.M.: Never Blame the Umpire - A Review of Situation Awareness Models and Methods for Examining the Performance of Officials in Sport Ergonomics, under review

12. Vicente, K.J.: Cognitive Work Analysis: Toward Safe, Productive, and Healthy ComputerBased Work. CRC Press, Boca Raton (1999)

13. Rasmussen, J.: Information Processing and Human-Machine Interaction. An Approach to Cognitive Engineering. North-Holland, New York (1986)

14. Read, G.J., Salmon, P.M., Lenné, M.G.: From work analysis to work design: A review of cognitive work analysis design applications. In: Proceedings of the Human Factors and Ergonomics Society Annual Meeting. SAGE Publications (2012)

15. Salmon, P.M., Cornelissen, M., Trotter, M.J.: Systems-based accident analysis methods: a comparison of Accimap, HFACS, and STAMP. Saf. Sci. 50(4), 1158-1170 (2012)

16. Stanton, N.A., et al.: Distributed situation awareness in dynamic systems: theoretical development and application of an ergonomics methodology. Ergonomics 49(12-13), 12881311 (2006)

17. Endsley, M.R.: Toward a theory of situation awareness in dynamic systems. Hum. Factors 37(1), 32-64 (1995) 
18. Salmon, P.M., et al.: What really is going on? Review of situation awareness models for individuals and teams. Theor. Issues Ergon. Sci. 9(4), 297-323 (2008)

19. Stanton, N.A., et al.: Is situation awareness all in the mind? Theor. Issues Ergon. Sci. 11(1-2), 29-40 (2009)

20. Smith, K., Hancock, P.A.: Situation awareness is adaptive, externally directed consciousness. Hum. Factors 37(1), 137-148 (1995)

21. Salmon, P.M., et al.: Distributed Situation Awareness: Theory, Measurement and Application to Teamwork. Ashgate, Aldershot (2009)

22. Hutchins, E.: How a cockpit remembers its speeds. Cogn. Sci. 19(3), 265-288 (1995)

23. Macquet, A.C., Stanton, N.A.: Do the coach and athlete have the same "picture" of the situation? Distributed situation awareness in an elite sport context. Appl. Ergon. 45(3), 724733 (2014)

24. De Keukelaere, C., et al.: Sharedness forms, contents and dynamics among expert sportteam. Formes, contenus et évolution du partage au d'une équipe de sport de haut niveau 76(3), 227-255 (2013)

25. Mallo, J., et al.: Effect of positioning on the accuracy of decision making of association football top-class referees and assistant referees during competitive matches. J. Sports Sci. 30(13), 1437-1445 (2012)

26. Ting, S., Chilukuri, M.: Novel pattern recognition technique for an intelligent cricket decision making system. In: Instrumentation and Measurement Technology Conference, 2009, I2MTC 2009. IEEE (2009)

27. Endsley, M.R.: Measurement of situation awareness in dynamic systems. Hum. Factors 37 (1), 65-84 (1995)

28. Salas, E., et al.: Situation awareness in team performance: implications for measurement and training. Hum. Factors 37(1), 123-136 (1995)

29. Australian Football League: Laws of Australian Football 2014. Australian Football League, Melbourne, Victoria (2014)

30. Marais, K., Dulac, N., Leveson, N.: Beyond normal accidents and high reliability organizations: the need for an alternative approach to safety in complex systems. In: Engineering Systems Division Symposium. MIT, Cambridge, March 2004

31. Salmon, P.M., et al.: The crash at Kerang: investigating systemic and psychological factors leading to unintentional non-compliance at rail level crossings. Accid. Anal. Prev. 50, 12781288 (2013)

32. Salmon, P.M., Walker, G., Stanton, N.: Broken components versus broken systems: why it is systems not people that lose situation awareness. Cogn. Technol. Work 17(2), 179-183 (2015)

33. Stanton, N.A., Salmon, P.M., Walker, G.H.: Let the reader decide a paradigm shift for situation awareness in sociotechnical systems. J. Cogn. Eng. Decis. Making 9(1), 44-50 (2015) 